\begin{tabular}{|c|c|c|}
\hline 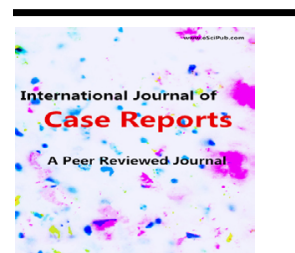 & $\begin{array}{l}\text { International Journal of Case Reports } \\
\text { (ISSN:2572-8776) }\end{array}$ & 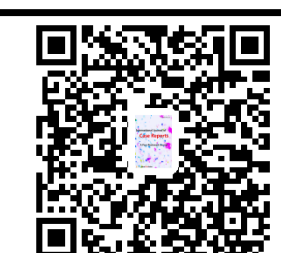 \\
\hline
\end{tabular}

\title{
Efficacy of an oral appliance in an adult Marfan syndrome patient with obstructive sleep apnea
}

\author{
Akifumi Enomoto*, Yuko Kinoshita, Atsushi-Doksa Lee, Miho Sukedai, Takeshi Shimoide \\ Department of Oral and Maxillofacial Surgery, Kindai University School of Medicine, 377-2, Ohno- \\ higashi, Osaka-Sayama, 589-8511, Japan.
}

\section{ABSTRACT}

Marfan's syndrome (MFS) is an autosomal dominant disorder that affects the body's connective tissue, characterized by changes in three major connective tissue systems: the musculoskeletal, ocular, and cardiovascular systems. Individuals with MFS can develop severe cardiovascular complications., and the life expectancy of patients with MFS is markedly reduced as the effects of the disease on the aorta often lead to progressive dilatation, with subsequent dissection and rupture. Obstructive sleep apnea (OSA) is highly prevalent in patients with cardiovascular diseases. There is a high prevalence of OSA in patients with MFS due to craniofacial dysmorphism and increased upper airway collapsibility. A high prevalence of OSA has been reported in patients with MFS. To date, however, there are only a few case reports regarding the treatment of OSA in MFS patients. We present a treatment in an adult MFS patient with OSA using an oral appliance (OA). The patient was a 48-year-old Japanese woman of MFS, diagnosed with moderate OSA (apnea hypopnea index $[\mathrm{AHI}]=27.0$; lowest oxygen saturation $[\mathrm{SaO} 2]=78 \%$; maximum apnea time $=114 \mathrm{~s}]$ ). An OA was selected as the treatment option, and post-treatment sleep study indicated a reduction in $\mathrm{AHI}$ to 8.8/h with an improvement in other factors (lowest $\mathrm{SaO} 2=84 \%$; maximum apnea time $=66 \mathrm{~s}$ ). Additionally, the treatment resulted in a reduction in BNP levels (81.3 from 110.2 pg/ $\mathrm{ml}$ ), and the patient's cardiac function was stable over the period in which the patient received OA treatment. This study provides a basis for the future appropriate understanding and management of OSA of MFS patients.

Keywords: OSA, oral appliance, Marfan's syndrome
${ }^{*}$ Correspondence to Author:

Akifumi Enomoto

Department of Oral and Maxillofacial Surgery, Kindai University School of Medicine, 377-2, Ohno-higashi, Osaka-Sayama, 589-8511, Japan.

How to cite this article:

Akifumi Enomoto, Yuko Kinoshita, Atsushi-Doksa Lee, Miho Sukedai, Takeshi Shimoide. Efficacy of an oral appliance in an adult Marfan syndrome patient with obstructive sleep apnea. International Journal of Case Reports, 2021 5:231

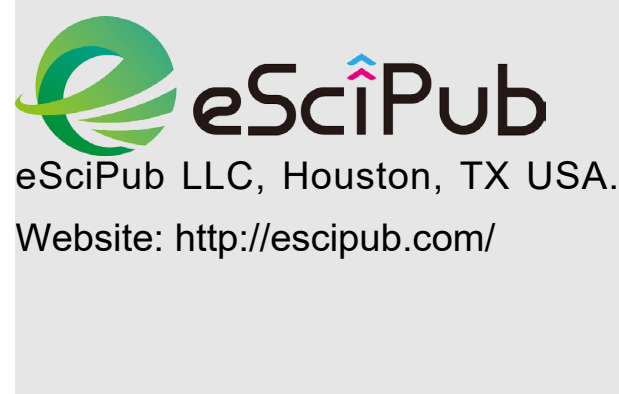




\section{Introduction}

Marfan's syndrome (MFS) is an autosomal dominant disorder that affects the body's connective tissue, characterized by changes in three major connective tissue systems: the musculoskeletal, ocular, and cardiovascular systems. MFS is caused by a mutation in the FBN1 gene, which causes changes in the protein, fibrillin, resulting in abnormal connective tissue with a wide range of manifestations.

Individuals with MFS can develop severe cardiovascular complications. The life expectancy of patients with MFS is markedly reduced as the effects of the disease on the aorta often lead to progressive dilatation, with subsequent dissection and rupture, despite medical and surgical advancements increasing the life span of MFS patients. Obstructive sleep apnea (OSA) is highly prevalent in patients with cardiovascular diseases, such as hypertension, coronary artery disease, and atrial fibrillation [1, 2] . Several studies subsequently proposed that hypoxemic stress caused by OSA could contribute to the pathogenesis of cardiac arrhythmias and conduction disturbances. The contribution of OSA in the pathophysiology of cardiovascular diseases has generated considerable importance. There is a high prevalence of OSA in patients with MFS due to craniofacial dysmorphism and increased upper airway collapsibility. Previously, a high prevalence of OSA has been reported in patients with MFS.
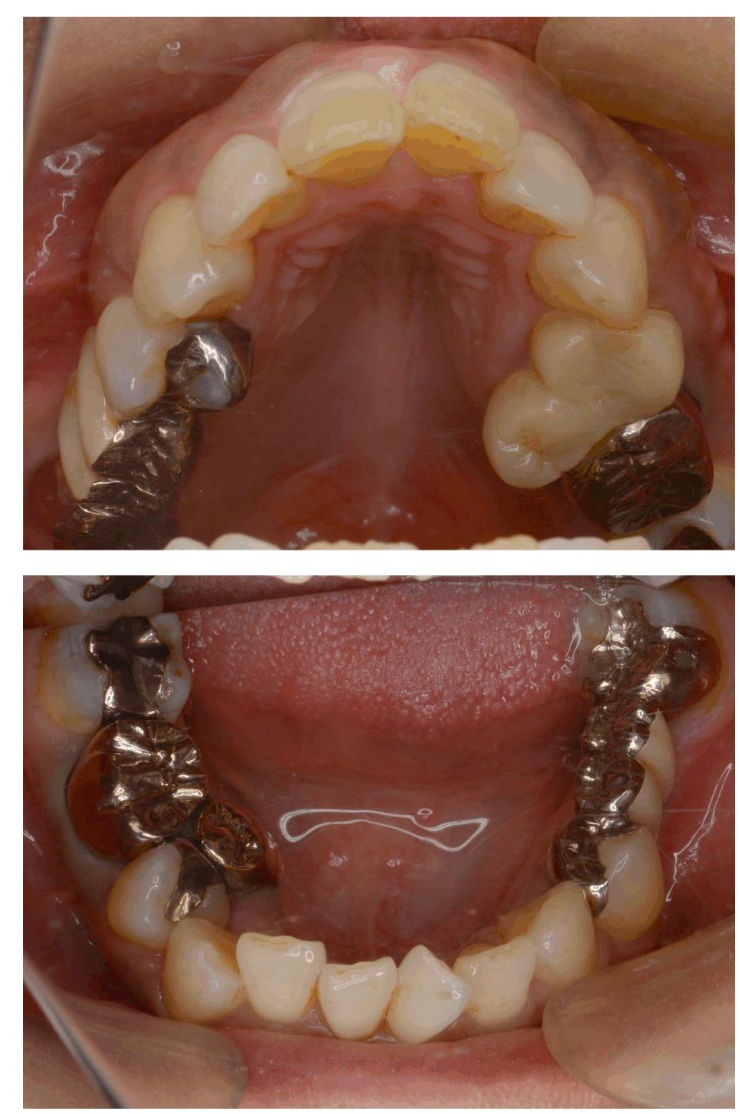

Fig. 1 Intraoral photographs of the patient's palate. A maxillary constriction and high arched palate were observed. 

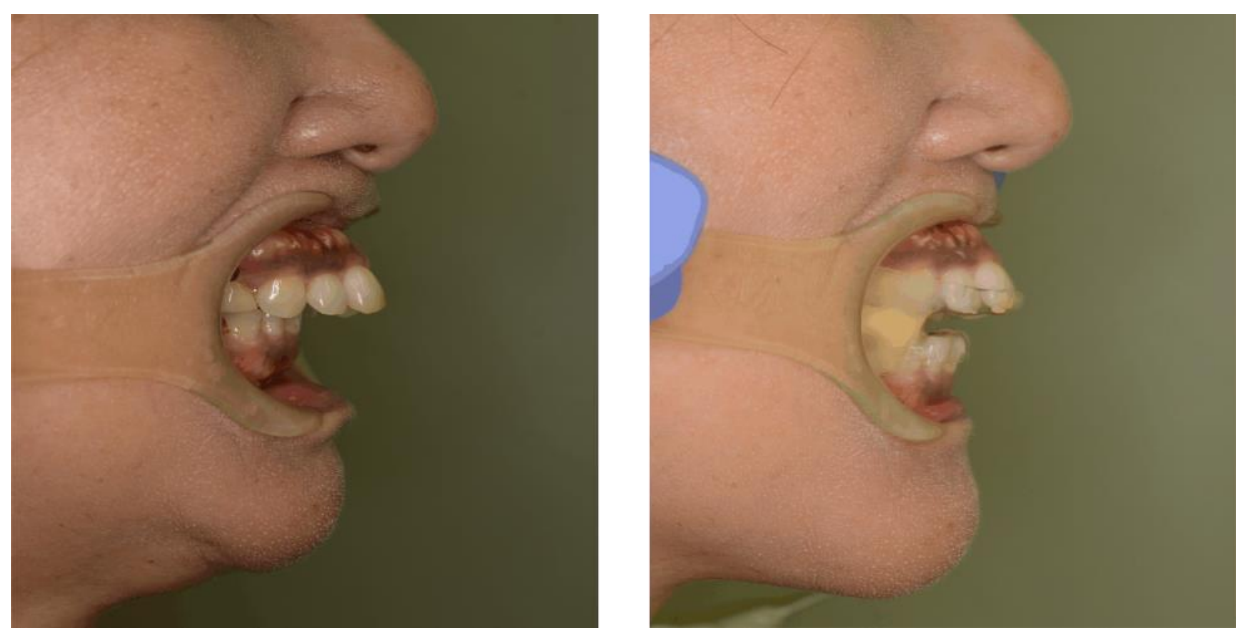

Fig. 2 Lateral extraoral photograph. The figure shows the lateral extraoral photograph without (left) and with (right) the OA.
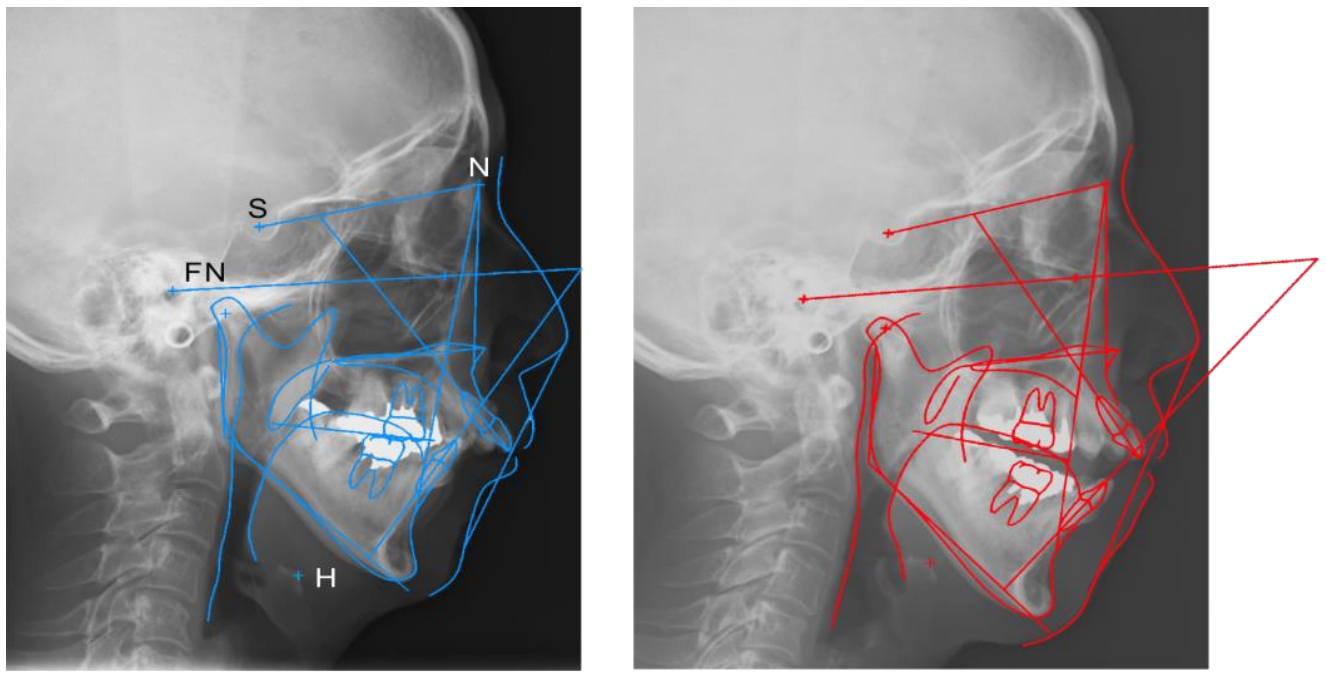

Fig. 3 Cephalometric analysis. The figure shows the cephalometric changes without (blue line, left) and with (red line, right) the OA. The following points were identified on lateral cephalograms: point $S$ (sella: the midpoint of the pituitary fossa), $N$ (nasion: the most anterior point on the frontonasal suture), FH (Frankfurt plane), and $\mathrm{H}$ (hyoidale).

To date, however, there are only a few reports regarding the treatment of OSA in MFS patients, using nasal continuous positive airway pressure (nCPAP) or a rapid maxillary expansion device [3]. To bridge the gap regarding the lack of information about OSA treatment in MFS patients, further studies are required. Here, we present a treatment in an adult MFS patient with OSA using an oral appliance (OA).

\section{Case report}

A 48-year-old Japanese woman was referred to our department in August 2017, complaining of the worsening of snoring. She was diagnosed with moderate OSA (apnea hypopnea index $[\mathrm{AHI}]=27.0 ;$ lowest oxygen saturation $[\mathrm{SaO} 2]=78 \%$; maximum apnea time $=114 \mathrm{~s}]$ ), and nCPAP was initiated as nCPAP has been adopted for treating patients with $\mathrm{AHI}>20$. IJCR: https://escipub.com/international-journal-of-case-reports/ 
However, she was unable to maintain CPAP treatment because of discomfort. Therefore, treatment with an OA was suggested by the doctor.

The patient was diagnosed with Marfan's syndrome at the age of 31 years. Physical examination revealed the typical body habitus of Marfan's syndrome; she was $175.5 \mathrm{~cm}$ tall with a body weight of $49.8 \mathrm{~kg}$. She had arachnodactyly and an arm span greater than her height. She had undergone vascular prosthesis replacement for aortic dissection in 1999 and the Bentall procedure for annuloaortic ectasia in 2013.

The patient's maxillary arch was crowded with a high palatal arch (Fig. 1). The patient's preoperative lateral cephalometric radiographs were obtained, and the radiographs were digitized, traced, and analyzed by a professional orthodontist. Clinically, she presented a Class II subdivision malocclusion associated with a severe anterior open bite (Fig. 2). Her major skeletal factors including SNA (sella, nasion, A point), SNB (sella, nasion, B point), and ANB (A point, nasion, $B$ point) were $75.9^{\circ}, 67.9^{\circ}$, and $8.0^{\circ}$, respectively. Her posterior airway space at different pharynx levels including superior posterior airway space (SPAS), middle airway space (MAS), and inferior airway space (IAS) were $10.2 \mathrm{~mm}, 12.6 \mathrm{~mm}$, and $13.8 \mathrm{~mm}$, respectively. Additionally, the distance between the mandibular plane and hyoid bone (MP-H) was $18.8 \mathrm{~mm}$ (table 1 and Fig.3). After consultation, an $\mathrm{OA}$ was selected as the treatment option after the initial examination.
She accepted OA treatment. A custom-made OA consisting of maxillary and mandibular components fixed by acrylic resin was used. The OA was fabricated at $70 \%$ of the maximum mandibular protrusion relative to the maximum intercuspation position (Fig. 2). The patient was instructed to use the OA every night. The cephalometric findings with and without the $\mathrm{OA}$ are shown in Figures 3. After OA treatment, the SNB and ANB angles changed to $68.6^{\circ}$ and $6.8^{\circ}$, respectively. Over jet dimensions changed to 8.5 $\mathrm{mm}$ from $17.5 \mathrm{~mm}$. Additionally, MP-H and SPAS dimensions changed to $11.4 \mathrm{~mm}$ and $21.5 \mathrm{~mm}$, respectively (Tale 1 ). The position of hyoid bone was elevated, and the pharyngeal airway space, especially the superior pharyngeal space, was widened by the treatment. After 2 years of OA treatment, the post-treatment sleep study indicated a reduction in $\mathrm{AHI}$ to $8.8 / \mathrm{h}$ with an improvement in other factors (lowest $\mathrm{SaO} 2=84 \%$; maximum apnea time $=66 \mathrm{~s}$ ). Additionally, the treatment resulted in a reduction in BNP levels. The patient's post-treatment BNP (human brain natriuretic peptide) level improved to 81.3 from $110.2 \mathrm{pg} / \mathrm{ml}$ after OA treatment. The patient's cardiac function was stable over the period in which the patient received $O A$ treatment. Additionally, the deoxyribonucleic acid (DNA) sequence analysis of FBN1 revealed a heterozygous missense pathogenic variant on FBN1: c.8302del1A (p.lle2768fs) in chr 15: 48411303. The variant is considered strongly pathogenic based on the American College of Medical Genetics classification. Genetic testing of the parents was not performed. Written, 
informed consent was obtained from the patient for publication of this study and any accompanying images.

\section{Discussion}

This study presents the description of the efficacy of an OA in an adult MFS patient with OSA.

The most serious complications in MFS involve the heart and aorta, with an increased risk of aortic dilatation and associated aortic dissection and rupture. Poor life expectancy in patients with MFS is mainly triggered by cardiovascular complications. Sleep disorders might be the pathological basis of cardiovascular diseases. OSA has deleterious effects on cardiovascular health ${ }^{[1,4]}$. There is a high prevalence of OSA in patients with MFS, possibly due to craniofacial dysmorphism and increased upper airway collapsibility. More than $30 \%$ of adult MFS patients exhibit OSA, which could contribute to cardiovascular morbidity. There is also preliminary evidence for a causal link between OSA and aortic dilatation in patients with MFS ${ }^{5-}$ 7].

It is possible that concomitant OSA in patients with MFS may have adverse effects, particularly with respect to aortic root dilatation. OSA may contribute to the pathophysiology of cardiovascular complication in MFS, and treatment of OSA with nCPAP is associated with a marked attenuation in the rate of aortic dilatation ${ }^{[5]}$. The several possible approaches for OSA prevention include CPAP devices, OA, orthodontic therapy, and surgical treatment.
Treatment should be considered depending on the compromised situation of each patient. Since our patient was an adult MFS patient with cardiovascular complications, orthodontic and/or surgical treatment was not indicated. Additionally, nCPAP was not adopted for the present patient because of discomfort. To date, there have been no reports on the efficacy of OAs for adult MFS patients with OSA. Only a few reports have described the effect of nCPAP in MFS patients. Another report showed that early rapid maxillary expansion and mandibular advancement using an OA improved the airway patency of children with MFS and described a possible preventive measure for OSA in MFS [3]. Recently, the involvement of other systems, including the skin, central nervous system, and respiratory system, has been recognized in MFS. MFS may increase the risk of pneumothorax because of the connective tissue changes caused by high air pressure applied to the lungs. It has been reported that the prevalence of pneumothorax in MFS is $4 \%-11 \%{ }^{[8]}$. Therefore, MFS patients are advised to avoid activities with high-pressure changes such as skydiving, playing wind instruments, or scuba diving [9]. nCPAP with long-term yearly usage may also increase the risk of this condition. Pulmonary symptoms are not a major feature of MFS, but spontaneous pneumothorax is common. In spontaneous unilateral pneumothorax, air escapes from the lung and occupies the pleural space between the chest wall and lung. The lungs become partially compressed or collapsed. This can cause pain, shortness of breath, cyanosis, and, if not treated, 
death.

The OA for OSA does not produce or increase these pulmonary manifestations. On the other hand, temporomandibular disorders and the risk of developing pain and functional impairment of the temporomandibular complex could be observed in OSA patients treated with an OA (mandibular advancement device), although these occur in the initial period of use [10]. MFS patients sometimes show a signs and symptoms of temporomandibular joint (TMJ) dysfunction, indicating symptoms of subluxation. However, findings related to the TMJ were not observed in the present patient 2 years after OA treatment. In the present patient, the soft tissue and upper airway were improved cephalometrically by OA treatment. As shown by the change in SPAS, MAS, and IAS data, the superior, middle, and inferior regions of the pharyngeal airway space were all widened by OA treatment. Thus, a reduction in respiratory resistance via mandibular advancement was achieved. This resulted in an improvement in the $\mathrm{AHI}$. As the AHI decreased, BNP levels also decreased. Thus, treatment of OSA with MFS is indicated to affect cardiovascular health. OA treatment for MFS with craniofacial dysmorphism and increased upper airway collapsibility could be a comprehensive treatment for cardiovascular diseases.

The issues associated with the treatment strategy for OSA with MFS have not been widely discussed. A better understanding of the mechanisms underlying cardiovascular complications of OSA will contribute to better recognition and treatment of these cardiovascular issues in MFS. Therefore, further research in this direction is required.

\section{Conflict of interest requirements}

The authors declare no conflict of interest.

\section{Funding}

No source of funding was used in this study.

\section{Ethical Approval}

$\mathrm{N} / \mathrm{A}$

\section{Acknowledgment}

We would like to express our sincere gratitude and feels immense pleasure in thanking to $\mathrm{Dr}$. Yoshihisa Takeuchi DDS and PhD for the orthodontic analysis.

\section{References}

[1]. Sampol, G., et al., Obstructive sleep apnea and thoracic aorta dissection. Am J Respir Crit Care Med, 2003. 168(12): p. 1528-31. 10.1164/rccm.200304-566OC.

[2]. Kyzer, S. and I. Charuzi, Obstructive sleep apnea in the obese. World J Surg, 1998. 22(9): p. 9981001.

[3]. Taddei, M., et al., Effects of rapid maxillary expansion and mandibular advancement on upper airways in Marfan's syndrome children: a home sleep study and cephalometric evaluation. Sleep Breath, 2015. 19(4): p. 1213-20. 10.1007/s11325-015-1141-y.

[4]. Saruhara, H., et al., Obstructive sleep apnea as a potential risk factor for aortic disease. Heart Vessels, 2012. 27(2): p. 166-73. 10.1007/s00380011-0135-3. 
[5]. Cistulli, P.A., et al., Aortic root dilatation in Marfan's syndrome: a contribution from obstructive sleep apnea? Chest, 1997. 111(6): p. 1763-6.

[6]. Kohler, M., et al., The prevalence of obstructive sleep apnoea and its association with aortic dilatation in Marfan's syndrome. Thorax, 2009. 64(2): p. 162-6. 10.1136/thx.2008.102756.

[7]. Verbraecken, J., et al., Aortic root diameter and nasal intermittent positive airway pressure treatment in Marfan's syndrome. Clin Genet, 2003. 63(2): p. 131-4.

[8]. Wood, J.R., et al., Pulmonary disease in patients with Marfan syndrome. Thorax, 1984. 39(10): p. $780-4$

[9]. von Kodolitsch, Y., et al., The role of the multidisciplinary health care team in the management of patients with Marfan syndrome. J Multidiscip Healthc, 2016. 9: p. 587-614. 10.2147/JMDH.S93680.

[10]. Doff, M.H., et al., Long-term oral appliance therapy in obstructive sleep apnea syndrome: a controlled study on temporomandibular side effects. Clin Oral Investig, 2012. 16(3): p. 689-97. 10.1007/s00784-0110555-6. 